of Pediatrics. University of California, San Diego, Medical Center. 225 Dickinson Strect. San Dicgo, California 91203

48. The authors are grateful to Dr. Augustin Gongora for his generous cooperation in obtaining the subjects' blood samples and to Dr. Ron Riggio for his help with the statistical analyses. This research was supported in part by a grant from the National Institutes of Health. HD 10111 and a contract with the
Agency for International Development. Department of State ta-C-1286.

49. This research was supported in part by a grant from the National Institutes of Health. HD 10111 and a contract with the Agency for International Development. Department of State, ta-C-1286.

50 Received for publication June $21,1982$.

51. Accepted for publication April 13,1983.

\title{
Complementation, Cross Correction, and Drug Correction Studies of Combined $\beta$-Galactosidase Neuraminidase Deficiency in Human Fibroblasts
}

\author{
PIETRO STRISCIUGLIO, KIM E. CREEK. AND WILLIAM S. SLY ${ }^{(17)}$ \\ The Edward Mallinckrodt Department of Pediatrics, Washington University School of Medicine, Division of \\ Medical Genetics, St. Louis Children's Hospital, St. Loulis, Missouri USA
}

\begin{abstract}
Summary
Neuraminidase activity in fibroblasts obtained from a patient with combined $\beta$-galactosidase-neuraminidase deficiency $(\beta$ $\mathrm{gal}^{-} /$neur') was partially restored by fusion with two ML I cell lines and an ML II cell line. As observed with neuraminidase activity, $\beta$-galactosidase also showed complementation with an increase in activity when $\beta$-gal ${ }^{-} /$neur $^{-}$fibroblasts were fused with an ML II or a $G_{M I}$ gangliosidosis cell line. Both $G_{M !}$ gangliosidosis and sialidosis fibroblasts secreted a "corrective factor" which, when added to medium above $\beta$-gal $/$ neur $^{-}$fibroblasts, was pinocytosed and partially corrected its deficiencies for these two enzymes. This partial correction of $\beta$-galactosidase and neuraminidase activities persisted for at least $72 \mathrm{~h}$ after removal of the "corrective factor" from the medium. A "corrective factor" with similar properties was obtained from glycoproteins isolated by chromatography of human spleen homogenates on concanavalin A-Sepharose. Treatment of $\beta$-gal $/ /$ neur $^{-}$fibroblasts with leupeptin or EP475, two inhibitors of lysosomal thiolproteases, partially restored $\beta$-galactosidase activity but caused no significant improvement in neuraminidase levels. The partial corrective effect of leupeptin on $\beta$-galactosidase activity persisted for at least $\mathbf{2} \mathbf{d}$ after removal of the drug, even in the presence of cycloheximide.
\end{abstract}

\section{Abbreviation}

$\beta$-gal ${ }^{-} /$neur ${ }^{-}, \beta$-galactosidase-neuraminidase deficiency

In the past few years, a defect in acid neuraminidase (sialidase) activity has been demonstrated in several disorders presenting various clinical phenotypes. Neuraminidase deficiency has also been demonstrated in another group of patients originally found to have a $\beta$-galactosidase deficiency $(14,16)$. In these cases the neuraminidase deficiency was inferred to be the primary metabolic block responsible for the $\beta$-galactosidase deficiency since $\beta$ galactosidase levels in the parents of affected patients were generally not reduced. Complementation analysis between cell lines obtained from patients deficient in both $\beta$-galactosidase and neuraminidase $\left(\beta\right.$-gal ${ }^{-} /$neur $\left.^{-}\right)$with those from patients affected only by neuraminidase deficiency (ML I) have been reported by two laboratories but with differing conclusions $(9,10)$. Using a heterokaryon enrichment procedure, Mueller and Shows (13) recently presented convincing evidence of restoration of neuraminidase activity after fusion of ML I fibroblasts with those from $\beta$-gal ${ }^{-} /$neur ${ }^{-}$. Furthermore, Hoogeveen et al. (8) showed that the combined deficiency of $\beta-\mathrm{gal}^{-} /$neur ${ }^{-}$in human fibroblasts in culture can be partially corrected by the addition of concentrated culture medium collected from several mutant lines of human fibroblasts, including those with isolated $\beta$-galactosidase $\left(\mathrm{G}_{\mathrm{M}}\right.$ gangliosidosis) or neuraminidase (ML I) deficiency. Similar corrective effects were observed by these authors by treating $\beta-\mathrm{gal}^{-} /$neur ${ }^{-}$fibroblasts with the protease inhibitor leupeptin (5). Suzuki et al. (15) were able to confirm this corrective effect with leupeptin for $\beta$-galactosidase but not neuraminidase.

In order to help clarify the metabolic defect responsible for the combined $\beta-\mathrm{gal}^{-} /$neur ${ }^{-}$deficiency we have conducted complementation, cross correction, and drug correction studies on fibroblasts obtained from a patient with the infantile form of combined $\beta$-gal ${ }^{-} /$neur $^{-}$that we have followed for the past $8 \mathrm{yr}$ (1). These studies provided additional support for the proposal recently made by d'Azzo et al. (3) that the combined $\beta$-gal ${ }^{-/}$ neur- deficiency is not due to a defect in the structural gene for $\beta$-galactosidase or neuraminidase, but rather in a glycoprotein which is normally required to protect these two enzymes against intralysosomal degradation.

\section{MATERIALS AND METHODS}

Materials. Sialyllactose was purchased from Sigma Chemical Co., St. Louis, MO. Most 4-methylumbelliferyl substrates were obtained from Research Products International Corp., Elk Grove Village, IL except the 4-methylumbelliferyl substrate for neuraminidase was from Kock-Light as was the polyethylene glycol (molecular weight 1000). Concanavalin A-Sepharose was from Pharmacia. Leupeptin and EP-475 were generous gifts from Dr. A. L. Goldberg, Dept. Physiology, Harvard Medical School, Boston, MA. Human spleens were obtained from the Dept. of 
Pathology, Washington University School of Medicine, St. Louis, MO. Cell lines from two patients with sialidase deficiency (ML I) (GM 2921 and GM 1718) and one patient with $\mathrm{G}_{\mathrm{M} \text { I }}$ gangliosidosis (GM 3589) were obtained from the Human Mutant Cell Repository, Camden, NJ. Fibroblasts from a patient (T. M.) with I-cell disease (ML II) were obtained from the Division of Medical Genetics, University of Texas Health Science Center, Dallas, TX. Cultured fibroblasts established by skin biopsy from our patient with combined $\beta$-gal ${ }^{-} /$neur ${ }^{-}$deficiency are available as line GM 0806 from the Human Mutant Cell Repository, Camden, NJ. They were listed in earlier catalogues as $\beta$-galactosidase deficient cells under $G_{M}$, gangliosidosis, because the neuraminidase deficiency was not appreciated initially.

Fibroblast Cultures. Cultures were maintained at $37^{\circ} \mathrm{C}$ in $5 \%$ $\mathrm{CO}_{2}$ in minimum essential medium (MEM Earle's medium, Gibco), supplementd with $15 \%$ heat-inactivated fetal calf serum (Gibco), $1 \mathrm{mM}$ sodium pyruvate, $100 \mathrm{U} / \mathrm{ml}$ penicillin, and 100 $\mu \mathrm{g} / \mathrm{ml}$ streptomycin sulfate. Cells were harvested 1-2 wk after subculture. Fibroblasts harvested from $100-\mathrm{mm}$ Petri dishes (Corning) were, after trypsinization, resuspended in either phosphate buffered saline or normal saline $(0.9 \%)$ and washed twice in the same solution by resedimentation in a clinical centrifuge. The cell pellet was then resuspended in $0.5 \mathrm{ml}$ of distilled water and homogenized in a Duall-type homogenizer with 10 strokes before enzymatic assavs and protein determinations. Fibroblasts harvested from $35-\mathrm{mm}$ Petri plates (Corning) were processed for protein and enzymatic assays as described above, except that the cells were washed with normal saline and then scraped from the plates without prior trypsinization. Fibroblasts used for "cross correction" studies were grown in 35-mm Petri dishes (Corning) at $37^{\circ} \mathrm{C}$ in minimum essential medium without bicarbonate, supplemented with $15 \%$ heat-inactivated fetal calf serum (Gibco), $1 \mathrm{mM}$ sodium pyruvate, $100 \mathrm{U} / \mathrm{ml}$ penicillin, $100 \mu \mathrm{g} /$ $\mathrm{ml}$ streptomycin sulfate, and $5 \%$ buffer consisting of $10 \mathrm{mM}$ sodium phosphate, $15 \mathrm{mM}$ BES, $10 \mathrm{mM}$ PIPES; $\mathrm{pH}$ 6.8, and $0.5 \%$ bicarbonate.

Collection of fibroblast secretions. Fibroblasts from the cell lines described above were grown to confluence in $490-\mathrm{cm}^{2}$ roller bottles (Corning). The growth medium was then replaced by 50 $\mathrm{ml}$ of serum-free Waymouth medium (KC Biological) containing $10 \mathrm{mM} \mathrm{NH}{ }_{4} \mathrm{Cl}$. The $\mathrm{NH}_{4} \mathrm{Cl}$ greatly enhances the secretion of "high-uptake" forms of acid hydrolases (7). The cells were maintained at $37^{\circ} \mathrm{C}$ for $24 \mathrm{~h}$ at which time the media was collected and replaced with $50 \mathrm{ml}$ of fresh media. The collections were continued for $1 \mathrm{wk}$ at which time the 7-24-h collections from each individual cell line were pooled, concentrated $(\sim 100$-fold $)$ and dialyzed against phosphate buffered saline on an Amicon PM 10 membrane filter.

Enzvmatic assavs and protein determination. Enzymatic activities were determined fluorometrically as previously described in part (6). Synthetic substrates used were 4-methylumbelliferyl- $\beta$ D-glucuronide, $10 \mathrm{mM}$ in $0.1 \mathrm{M}$ sodium acetate buffer, $\mathrm{pH} 4.8$, for $\beta$-glucuronidase activity; 4 -methylumbelliferyl- $\alpha$-D-mannopyranoside, $1 \mathrm{mM}$ in $0.15 \mathrm{M}$ citrate buffer, $\mathrm{pH} 4.0$, for $\alpha$ mannosidase activity; 4 -methylumbelliferyl- $\beta$-D-galactopyrano-

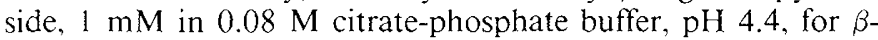
galactosidase activity; 4-methylumbelliferyl- $\alpha$-D-galactoside, 5

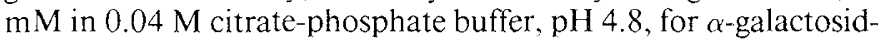
ase activity; 4-methylumbelliferyl- $\alpha$-L-fucoside, $0.96 \mathrm{mM}$ in 0.1 $\mathrm{M}$ citrate-phosphate buffer, $\mathrm{pH} 4.5$, for $\alpha$-fucosidase activity; 4 methylumbelliferyl-2-acetamido-2-deoxy- $\beta$-D-glucopyranoside, $5 \mathrm{mM}$ in $12 \mathrm{mM}$ citrate and $20 \mathrm{mM}$ phosphate buffer, $\mathrm{pH} \mathrm{4.4}$, for $\beta$-hexosaminidase activity; 4 -methylumbelliferyl- $\alpha$-D-gluco-

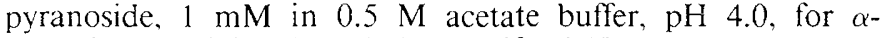
glucosidase activity: 4-methylumbelliferyl- $N$-acetyl- $\alpha$-D-neuramide, $4 \mathrm{mM}$ in $0.1 \mathrm{M}$ acetate buffer, $\mathrm{pH} 4.4$, for $\alpha$-neuraminidase activity. Assays were initiated by the addition of $25 \mu \mathrm{l}$ of cell homogenate $(10-100 \mu \mathrm{g}$ protein) to $100 \mu \mathrm{l}$ of substrate and incubated at $37^{\circ} \mathrm{C}$ for $30-60 \mathrm{~min}$, except $\alpha$-neuraminidase activity was measured by incubating $10 \mu \mathrm{l}$ of cell homogenate with
$30 \mu \mathrm{l}$ of $0.1 \mathrm{M}$ acetate buffer, $\mathrm{pH} 4.4$ and $15 \mu \mathrm{l}$ of fluorometric substrate. Reactions were stopped by the addition of $1.8 \mathrm{ml}$ of glycine carbonate buffer, $\mathrm{pH}$ 10.5. All assays were performed in duplicate. One unit of enzyme activity is defined as the amount of enzyme that releases $1 \mathrm{nmol}$ of 4-methylumbelliferone per $\mathrm{h}$.

$\alpha$-Neuraminidase activity was also measured using [ $\left.{ }^{3} \mathrm{H}\right]$ labeled sialyllactose as the substrate by the method described by Frisch and Neufeld (4) except no attempt to separate the $2 \rightarrow 3$ and 2 $\rightarrow 6$ isomers of the $\left[{ }^{3} \mathrm{H}\right]$ sialyllactitol was made. When using this substrate the enzymatic activity was calculated as the percentage of substrate hydrolyzed per h per mg cell protein. Protein was estimated by the method of Lowry et al. (11) using bovine serum albumin as the standard.

Complementation studies. Fibroblasts for complementation analysis were seeded at a ratio of $1: 1$ with each parental line and cultivated for $3 \mathrm{~d}$ in minimal essential medium (MEM Earle's medium, Gibco), supplemented with $15 \%$ heat-inactivated fetal calf serum (Gibco), $1 \mathrm{mM}$ sodium pyruvate, $100 \mathrm{U} / \mathrm{ml}$ penicillin, and $100 \mu \mathrm{g} / \mathrm{ml}$ streptomycin sulfate. Hybridization was then carried out by the addition of $42 \%(\mathrm{w} / \mathrm{v})$ polyethylene glycol 1000 as described by Hoogeveen et al. (9).

Cross correction studies. We studied the effects of "corrective factor" isolated from two sources on the activities of $\beta$-galactosidase and $\alpha$-neuraminidase in $\beta$-gal ${ }^{-} /$neur $^{-}$fibroblasts and other mutant cell lines. In one case we added $100 \mu \mathrm{l}$ of concentrated secretions prepared from human fibroblasts (as described above) to $1.0 \mathrm{ml}$ of medium which was then added to different mutant cell lines for $12 \mathrm{~h}$ to $3 \mathrm{~d}$. $\beta$-Galactosidase and $\alpha$-neuraminidase activities in these cells were measured at the times indicated in the figures. Correction was also studied by the addition of concanavalin A binding glycoproteins isolated from human spleen with modifications of a procedure described earlier (12). Human spleen was minced with a razor blade and then homogenized with a Polytron in 5 volumes of buffer $\mathrm{A}(0.025 \mathrm{M}$ sodium tartrate, $0.025 \mathrm{M}$ EDTA, pH 6.3). Deoxycholate (1\%) was then added to a final concentration of $0.2 \%$, the preparation homogenized a second time with a Polytron, and placed on ice for 30 $\mathrm{min}$. The crude homogenate was centrifuged for $20 \mathrm{~min}$ at 10,000 $\times g$, the supernatant removed, the pellet re-extracted in a small volume of buffer $\mathrm{A}$, and centrifuged again. The supernatant from the second extraction was combined with the first and solid ammonium sulfate was added to a final concentration of $55 \%$. The preparation was stirred for $45 \mathrm{~min}$ at $4^{\circ} \mathrm{C}$ and the pellet collected by centrifugation for $30 \mathrm{~min}$ at $10,000 \times \mathrm{g}$. The pellet was resuspended and dialyzed against buffer $\mathrm{B}$ ( $10 \mathrm{mM}$ Tris, 10 $\mathrm{mM} \mathrm{NaH} \mathrm{PO}_{4}, 100 \mathrm{mM} \mathrm{NaCl}, \mathrm{pH}$ 7.5). The dialyzed sample was applied to a column of concanavalin A-Sepharose $5.0 \mathrm{~m}$ of packed beads) equilibrated with buffer $\mathrm{B}$ at $4^{\circ} \mathrm{C}$. The column was then washed with 10 column volumes of buffer $B$ and eluted at room temperature with 7 column volumes of buffer B containng $0.75 \mathrm{M} \alpha$-methylmannoside. The eluate was dialyzed against buffer B and concentrated on an Amicon PM 10 filter to a final protein concentration of $11 \mathrm{mg} / \mathrm{ml}$. An aliquot $(50 \mu \mathrm{l})$ of this preparation was added to $1.0 \mathrm{ml}$ of medium which was then incubated with different mutant cell lines for $12 \mathrm{~h}$ to $3 \mathrm{~d}$.

\section{RESULTS}

Complementation studies. To study the genetics of our patient with the infantile form of combined $\alpha$-galactosidase-neuraminidase deficiency $\left(\beta-\mathrm{gal}^{-} /\right.$neur $\left.^{-}\right)$we have conducted complementation analysis by somatic cell hybridization to produce heterokaryons. Each of the parental cell types used in the complementation studies were deficient in $\alpha$-neuraminidase, $\beta$-galactosidase or both activities. Neuraminidase activity in fibroblasts obtained from the $\beta-\mathrm{gal}^{-} /$neur ${ }^{-}$patient was partially restored by fusion with two ML I cell lines (Table 1). After fusion, neuraminidase activity in the heterokaryons was restored to $9-16 \%$ of mean levels for control fibroblasts. Complementation was also observed following fusion with an ML II cell line. Here activity of 
Table 1. Complementation of $\beta$-gal-/neur fibroblasts with other neuraminidase deficient cell lines

\begin{tabular}{|c|c|c|c|c|}
\hline \multirow[b]{2}{*}{ Fusion } & \multicolumn{4}{|c|}{$\alpha$-Neuraminidase activity* } \\
\hline & Parental & $\begin{array}{l}\text { Parental } \\
\text { fusion }\end{array}$ & Co-cultivated & Fused \\
\hline$\overline{\beta-\mathrm{gal}^{-} / \text {neur }^{-}}$ & 2.3 & 2.5 & 2.3 & 8.1 \\
\hline $\mathrm{XMLI}$ & 0.6 & 1.9 & & \\
\hline$\beta$-gal ${ }^{-} /$ncur $^{-}$ & 2.3 & 2.7 & 2.4 & 13.8 \\
\hline$X \mathrm{ML} \mathrm{I}+$ & 0.6 & 1.4 & & \\
\hline$\beta-\mathrm{gal}^{-} / \mathrm{neur}^{-}$ & 2.3 & 3.6 & 3.8 & 19.4 \\
\hline X ML II & 0.5 & 1.5 & & \\
\hline
\end{tabular}

* Neuraminidase activities are expressed as nmol of 4-methylumbelliferyl- $N$-acetyl-neuraminide cleaved per $h$ per $\mathrm{mg}$ cell protein. Mean activity of controls are 88 (range $51-118$ ).

† Cell line GM 2921.

$\uparrow$ Cell line GM 1718

Table 2. Complementation of $\beta$-gal $/$ neur fibroblasts with a ML II cell line and a $\beta$-galactosidase deficient cell line

\begin{tabular}{|c|c|c|c|c|}
\hline \multirow[b]{2}{*}{ Fusion } & \multicolumn{4}{|c|}{$\beta$-Galactosidase activity* } \\
\hline & Parental & $\begin{array}{l}\text { Parental } \\
\text { fusion }\end{array}$ & Co-cultivation & Fused \\
\hline$\beta$-gal $/$ neur $^{-}$ & 156 & 118 & 132 & 302 \\
\hline X ML II & 14 & 13 & & \\
\hline$\beta-$ gal $^{-} /$neur $^{-}$ & 156 & 102 & 117 & 461 \\
\hline $\begin{array}{l}X \mathrm{G}_{\mathrm{M} \text { I gan- }} \\
\text { gliosidosis }\end{array}$ & 3 & 6 & & \\
\hline
\end{tabular}

* $\beta$-Galactosidase activities are expressed as nmol of 4-methylumbelliferyl-3-1)-galactopyranoside cleaved per $\mathrm{h}$ per $\mathrm{mg}$ cell protein. Mean activity of controls are 1751 (range 1150-2300).

neuraminidase reached $22 \%$ of mean control levels after fusion (Table 1). No increase in neuraminidase levels was observed following parental fusion.

We also tested the $\beta$-gal ${ }^{-} /$neur ${ }^{-}$fibroblasts for complementation with regard to $\beta$-galactosidase by fusion with ML II fibroblasts and fibroblasts from a $G_{M}$ gangliosidosis cell line. As observed with neuraminidase activity, $\beta$-galactosidase showed complementation with an increase in activity when fused with the ML II cell line (17\% of mean control levels) and the $G_{M}$ gangliosidosis fibroblasts ( $26 \%$ of mean control levels) (Table 2). These results suggest that the gene mutation in our $\beta-\mathrm{gal}^{-} /$neur $^{-}$ patient is different from the mutations observed in ML I, ML II, or $\mathrm{G}_{\mathrm{V},}$ gangliosidosis.

The small increase in neuraminidase (Table 1) and $\beta$-galactosidase (Table 2) levels we observed during co-cultivation may indicate the uptake by the $\beta$-gal ${ }^{-} /$neur ${ }^{-}$fibroblasts of a "corrective factor" secreted by the $\mathrm{G}_{\mathrm{x}_{1}}$ gangliosidosis or ML I fibroblasts. Similarly, in co-cultivation studies reported by Hoogeveen et al. (9), neuraminidase activity increased $5-7$-fold in $\beta-\mathrm{gal}^{-} / \mathrm{neur}^{-}$ cells as a result of the transfer of a "corrective factor" secreted by ML I fibroblasts. The small increase in neuraminidase and $\beta$ galactosidase activities we observed during co-cultivation of $\beta$ $\mathrm{gal}^{-} /$neur ${ }^{-1}$ fibroblasts with ML II fibroblasts is unexpected because secretions from ML II fibroblasts fail to correct $\beta$-gal ${ }^{-} /$ neur ${ }^{-}$fibroblasts (9).

Cross correction studies. Both $\mathrm{G}_{\mathrm{M}}$ gangliosidosis and sialidosis fibroblasts produced a "corrective factor" which was pinocytosed by the $\beta$-gal ${ }^{-} /$neur $^{-}$fibroblasts leading to partial correction of its deficiencies for these two enzymes. Concentrated secretions produced by a $G_{M 1}$ gangliosidosis cell line in the presence of $\mathrm{NH}_{4} \mathrm{Cl}$ were added to the medium above $\beta-\mathrm{gal}^{-} /$neur fibroblasts. A time dependent correction of $\beta$-galactosidase $(23 \%$ of mean control levels) and neuraminidase activities ( $11 \%$ of mean con-

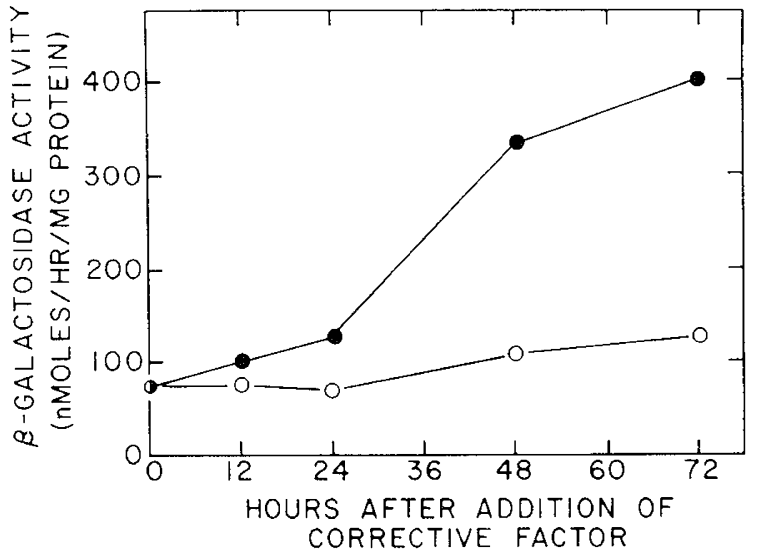

Fig. 1. Partial correction of $\beta$-galactosidase activity in $\beta$-gal ${ }^{-} /$neur $^{-}$ fibroblasts after addition of fibroblast secretion "corrective factor." $\mathrm{Fi}$ broblasts were grown to confluence in $35-\mathrm{mm}$ Petri dishes. At time zero, $100 \mu \mathrm{l}$ of concentrated secretions collected from $G_{M}$ gangliosidosis fibroblasts were added to each plate in $1.0 \mathrm{ml}$ of medium in the absence - or presence $(O)$ of $2 \mathrm{mM}$ mannose-6-phosphate. At the times indicated $\beta$-galactosidase activity was determined in duplicate plates.

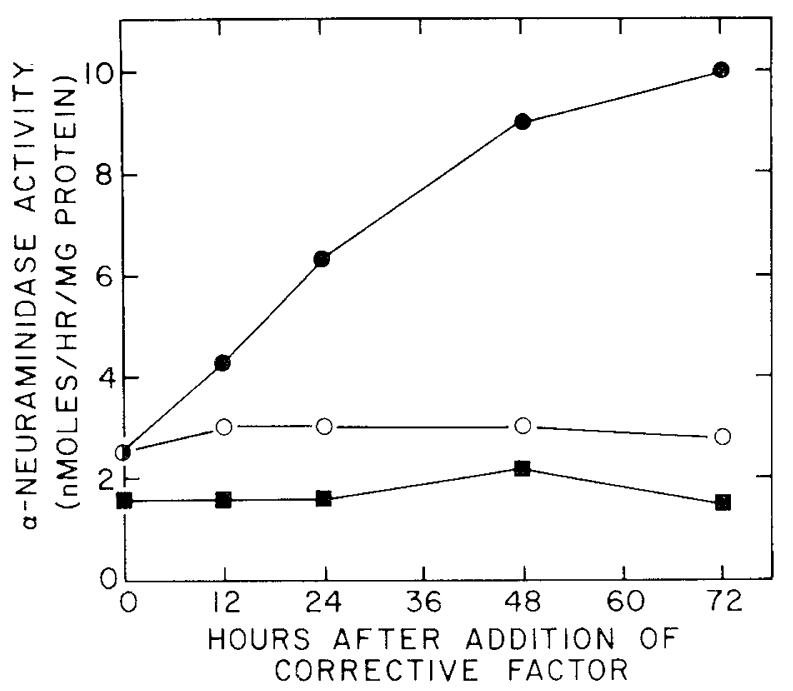

Fig. 2. Partial correction of $\alpha$-neuraminidase activity in $\beta-\mathrm{gal}^{-} / \mathrm{neur}^{-}$ fibroblasts after addition of fibroblast secretion "corrective factor." Fibroblasts were grown to confluence in $35-\mathrm{mm}$ Petri dishes. At time zero, $100 \mu$ l of concentrated secretions collected from $\mathrm{G}_{\mathrm{M} 1}$ gangliosidosis fibroblasts were added to $\beta$-gal ${ }^{-} /$neur $^{-}$fibroblasts in $1.0 \mathrm{ml}$ of medium in the absence ( ) or presence $(O)$ of $2 \mathrm{mM}$ mannose-6-phosphate or to ML I fibroblasts without added mannose 6-phosphate (1). At the times indicated $\alpha$-neuraminidase activity was determined in duplicate plates.

trol levels) were observed for the $\beta$-gal ${ }^{-} /$neur $^{-}$cells but no correction of neuraminidase was observed in control ML I cells (Figs. 1 and 2). Identical results were observed when secretions collected from ML I fibroblasts were used as the source of "corrective factor." Here $\beta$-gal ${ }^{-} /$neur $^{-}$fibroblasts displayed partial correction of neuraminidase and $\beta$-galactosidase activities whereas $G_{M 1}$ gangliosidosis controls showed no increase in $\beta$ galactosidase activity (data not shown). The secretions collected from the $\beta-\mathrm{gal}^{-} /$neur ${ }^{-}$fibroblasts had no corrective activity. The presence of mannose- 6 -phosphate $(2 \mathrm{mM})$ in the medium during incubations with "corrective factor" inhibited the partial correction of $\beta$-galactosidase and neuraminidase activities in the $\beta$ $\mathrm{gal}^{-} /$neur ${ }^{-}$cells (Figs. 1 and 2 ). This result suggests that the "corrective factor" is pinocytosed via the phosphomannosyl receptor present on fibroblasts, the receptor responsible for the 
uptake of many acid hydrolases. Furthermore, this partial correction of $\beta$-galactosidase and neuraminidase activities persists for at least $72 \mathrm{~h}$ after the removal of "corrective factor" from the medium of the $\beta$-gal ${ }^{-} /$neur $^{-}$fibroblasts (Fig. 3).

A "corrective factor" with properties similar to those observed for the factor from fibroblast secretions was obtained from glycoproteins isolated by chromatography of human spleen homogenates on concanavalin A-Sepharose. When these glycoproteins were added to the medium above $\beta$-gal ${ }^{-} /$neur $^{-}$cells, $\beta$-galactosidase activity increased to $13 \%$ of mean control levels and neuraminidase activity to $11 \%$ of mean control levels (Fig. 4). This increase is not due to the uptake of neuraminidase or $\beta$ galactosidase present in the spleen glycoprotein preparation because the levels of these enzymes did not increase after incubations of the spleen "corrective factor" with either ML I or $G_{M}$ I gangliosidosis fibroblasts (not shown). As with the "corrective factor" obtained from fibroblast secretions, the partial correction of $\beta$-galactosidase and neuraminidase activities persists for at least $72 \mathrm{~h}$ after removal of the spleen glycoprotein "corrective

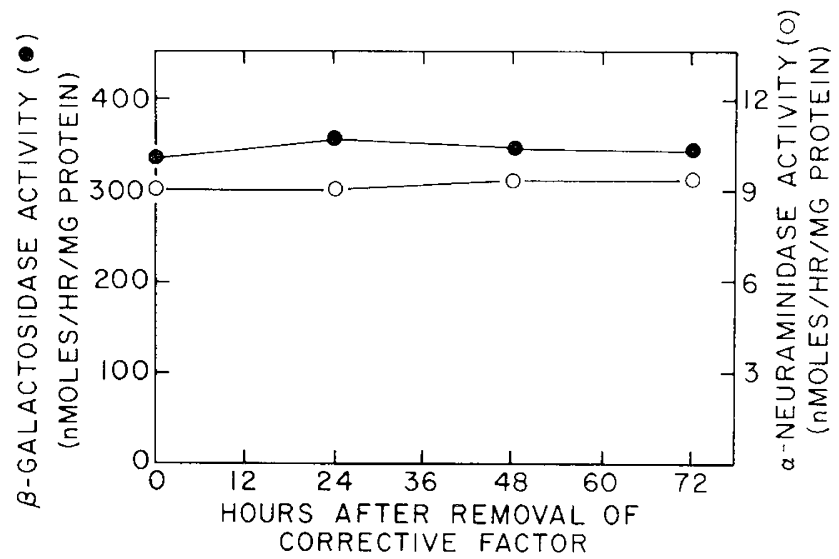

Fig. 3. $\beta$-Galactosidase and $\alpha$-neuraminidase activities in $\beta$-gal ${ }^{-} /$ neur ${ }^{-}$fibroblasts after removal of "corrective factor." $\beta$-gal ${ }^{-} /$neur $^{-}$fibroblasts were incubated for 2 days in $1.0 \mathrm{ml}$ of medium containing $100 \mu \mathrm{l}$ of concentrated secretions collected from $\mathrm{G}_{\mathrm{M} 1}$ gangliosidosis fibroblasts. At time zero this medium was preplaced by $1.0 \mathrm{ml}$ of fresh medium without "corrective factor" and at the times indicated $\beta$-galactosidase and $(\alpha$-neuraminidase $(O)$ ativities were determined in dulicate plates.

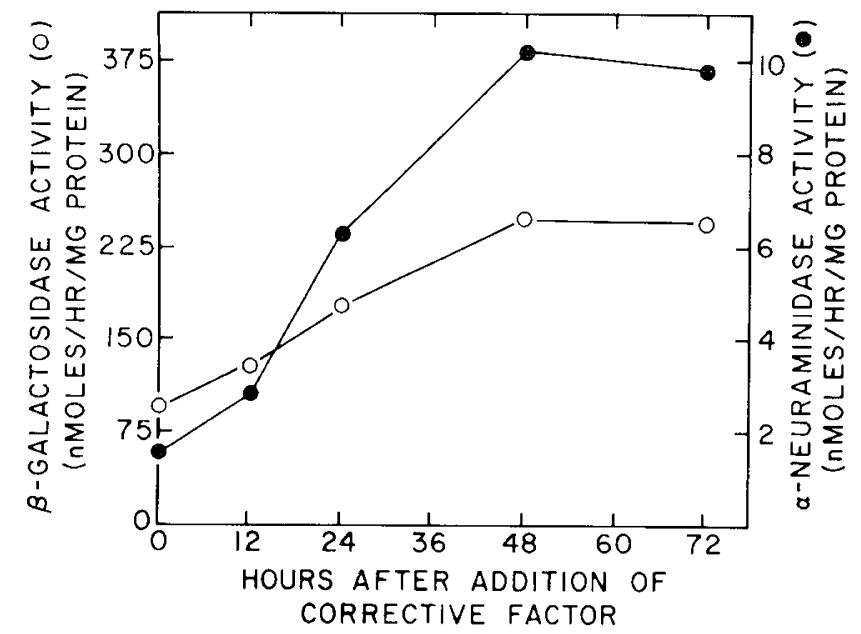

Fig. 4. Partial correction of $\beta$-galactosidase and $\alpha$-neuraminidase activity in $\beta$-gal ${ }^{-} /$neur $^{-}$fibroblasts after addition of "corrective factor" isolated from human spleen. Fibroblasts were grown to confluence in 35- $\mathrm{mm}$ Petri dishes. At time zero, $50 \mu \mathrm{l}$ of glycoproteins isolated from human spleen were added to $\beta$-gal ${ }^{-} /$neur ${ }^{-}$fibroblasts. At the times indicated $\beta$-galactosidase $(O)$ and $\alpha$-neuraminidase (๑) activities were determined in duplicate plates.
Table 3. Effect of protease inhibitors on acid hydrolase activities in $\beta$-gal $/$ neur fibroblasts

\begin{tabular}{lrcc}
\hline & \multicolumn{3}{c}{ Additions $\dagger$} \\
\cline { 2 - 4 } \multicolumn{1}{c}{ Acid hydrolase* } & None & Leupeptint & EP475 $\S$ \\
\hline$\beta$-galactosidase & 156 & 533 & 362 \\
$\alpha$-neuraminidase & 7 & 11 & 9 \\
$\alpha$-fucosidase & 60 & 58 & 64 \\
$\alpha$-glucosidase & 27 & 27 & 27 \\
$\alpha$-mannosidase & 69 & 63 & 71 \\
$\beta$-hexosaminidase & 9525 & 10563 & 10022 \\
$\alpha$-galactosidase & 67 & 67 & 69 \\
$\beta$-glucuronidase & 288 & 311 & 300 \\
\hline
\end{tabular}

* All enzyme activities except $\alpha$-neuraminidase were measured with the fluorometric substrates described in "Materials and Methods" and are expressed as nmol of substrate cleaved per h per $\mathrm{mg}$ cell protein. $\alpha-$ Neuraminidase activity was measured with $\left[{ }^{3} \mathrm{H}\right]$ sialyllactitol and is expressed as percentage substrate hydrolyzed per $\mathrm{h}$ per $\mathrm{mg}$ cell protein. Control enzyme activities are 1751 (range 1150-2300) and 95 (range 79114) for $\beta$-galactosidase and neuraminidase respectively.

$\dagger \beta$-gal ${ }^{-} /$neur ${ }^{-}$fibroblasts were grown to confluence, incubated in the presence or absence of inhibitors for $7 \mathrm{~d}$, and enzymatic activities determined.

F Leupeptin at a final concentration of $30 \mu \mathrm{M}$ was dissolved directly into growth medium.

$\S$ EP475 at a final concentration of $20 \mu \mathrm{M}$ was soluble in the growth medium when neutralized by the addition of an equimolar amount of $\mathrm{NaHCO}_{3}$.

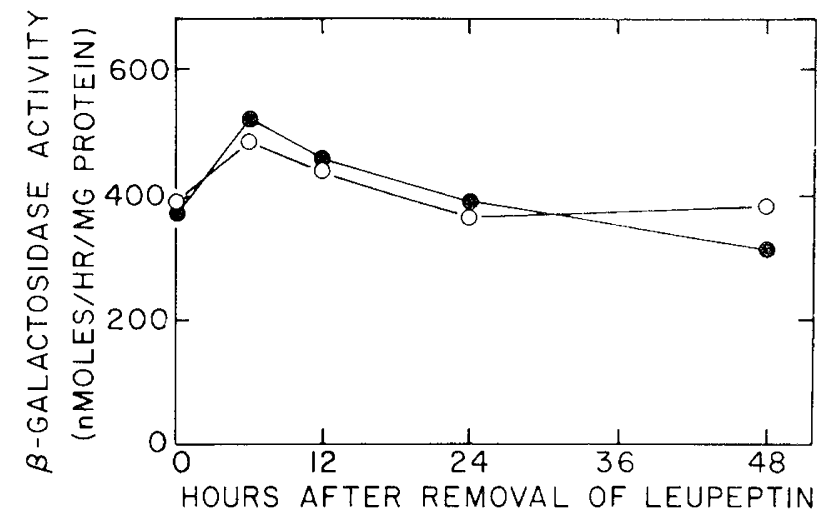

Fig. 5. $\beta$-Galactosidase activity in $\beta$-gal ${ }^{-} /$ncur $^{-}$fibroblasts after removal of leupeptin. $\beta$-gal ${ }^{-} /$neur $^{-}$fibroblasts were grown in $35-\mathrm{mm}$ Petri dishes for $1 \mathrm{wk}$ in the presence of $30 \mu \mathrm{M}$ leupeptin. At time zero this medium was replaced with $1.0 \mathrm{ml}$ of fresh medium in the absence or the presence $(O)$ of $10 \mu \mathrm{M}$ cycloheximide. At the times indicated $\beta$ galactosidase activity was determined in duplicate plates.

factor" from the medium of the $\beta-\mathrm{gal}^{-} /$neur ${ }^{-}$fibroblasts. The results (data not shown) were identical to those shown in Figure 3 for fibroblast secretion "corrective factor."

Drug correction studies. Treatment of $\beta$-gal ${ }^{-} /$neur ${ }^{-}$fibroblasts with leupeptin or EP 475, two inhibitors of lysosomal proteases, partially restored $\beta$-galactosidase activity. If $\beta$-gal ${ }^{-} /$neur $^{-}$fibroblasts were incubated with leupeptin for $7 \mathrm{~d} \beta$-galactosidase activity increased to $30 \%$ of mean control levels while with EP 475 it reached $21 \%$ of normal levels (Table 3 ). Unlike the effects seen with "corrective factor" from either concentrated fibroblasts secretions or glycoproteins isolated from human spleen these protease inhibitors caused no significant improvement of neuraminidase levels. Furthermore, they had no effect on the activities of several other acid hydrolases (Table 3). Addition of leupeptin to ML I or $\mathrm{G}_{\mathrm{M} \text { I }}$ gangliosidosis fibroblasts had no effect on the activities of neuraminidase, $\beta$-galactosidase, or the activities of the other acid hydrolases measured in Table 3 (data not shown). The partial corrective effect of leupeptin on $\beta$-galacto- 
sidase activity persisted for at least $2 \mathrm{~d}$ after removal of the drug, even in the presence of cycloheximide (Fig. 5). This result suggests that the sustained increase in $\beta$-galactosidase activity after removal of leupeptin is not dependent on new protein synthesis. Apparently, $\beta$-galactosidase accumulated in the presence of leupeptin is stable after its removal.

\section{DISCUSSION}

The clinical features of this American case of infantile combined $\beta$-galactosidase/neuraminidase deficiency have been summarized elsewhere (1), together with those of a similar Italian patient whose fibroblasts were studied by Galjaard et al. (5). Our findings that partial restoration of neuraminidase activity occurs after fusion of $\beta$-gal ${ }^{-} /$neur ${ }^{-}$fibroblasts with two ML I cell lines and an ML II cell line indicate that separate gene mutations are responsible for these diseases. Our results support those in a recently published report by Mueller and Shows (13), who used a heterokaryon enrichment procedure, and obtained an increase in neuraminidase activity that approached control values. Hoogeveen et al. (9) using fusions similar to ours, also found moderate increases in neuraminidase activity; however, Kato et al. (10) failed to demonstrate detectable increases in neuraminidase activity. The reasons for the discrepancy of Kato's findings with the other studies including this one are not clear. The restoration of $\beta$-galactosidase activity after fusion of $G_{M 1}$ gangliosidosis or ML II cells with $\beta$-gal ${ }^{-} /$neur $^{-}$fibroblasts suggests that different gene mutations, leading to the impairment of $\beta$-galactosidase, are involved in these disorders.

The partial correction of $\beta$-galactosidase and neuraminidase activities observed in $\beta$-gal ${ }^{-} /$neur ${ }^{-}$fibroblasts after the addition of concentrates of culture medium obtained from $\mathrm{G}_{\mathrm{M} \text { 1 }}$ gangliosidosis or ML I fibroblasts also supports our conclusions obtained from the complementation studies. Specifically, that the metabolic defect in the $\beta$-gal $/$ neur ${ }^{-}$is different from the basic defect responsible for $G_{M}$ gangliosidosis or sialidosis. Concentrated medium obtained from $\beta$-gal ${ }^{-} /$neur ${ }^{-}$fibroblasts is unable to correct itself. Cross correction studies published recently by Hoogeveen et al. (8) reached conclusions similar to ours.

The ability of mannose-6-phosphate to inhibit the partial correction of $\beta$-galactosidase and neuraminidase activities when media concentrates were added to $\beta-\mathrm{gal}^{-} /$neur ${ }^{-}$cells suggest that the "corrective factor" is being taken up by the fibroblasts via phosphomannosyl receptors present on the cell surface. These are the receptors responsible for the uptake of a variety of acid hydrolases by fibroblasts in culture. This suggests that the "corrective factor" could be an acid hydrolase itself or some other phosphoglycoprotein necessary for full enzymatic expression of $\beta$-galactosidase and neuraminidase. This conclusion agrees with the observation that medium collected from I-cells, which are unable to phosphorylate their acid hydrolases, does not correct $\beta$-gal ${ }^{-} /$neur ${ }^{-}$fibroblasts (9). The fact that the partial correction of $\beta$-galactosidase and neuraminidase activities persists for at least $3 \mathrm{~d}$ after removal of the "corrective factor" supports the argument that it may be important in stabilizing these two enzymes.

Another rich source of "corrective factor" was found to be glycoproteins isolated by chromatography of human spleen homogenates on concanavalin A-Sepharose. Glycoprotein preparations made in a similar manner from human placenta or human liver were effective in partially correcting neuraminidase levels but were ineffective in the correction of $\beta$-galactosidase activity (8). The apparent discrepancy between our results with spleen and those reported by others (8) with liver or placenta can be explained by the fact that spleen contains a large population of platelets, a rich source of "high uptake" phosphoglycoproteins that are pinocytosed by the phosphomannosyl recognition system present on fibroblasts (2).

We found that leupeptin or EP475, two thiol-protease inhibitors, were able to partially correct the $\beta$-galactosidase deficiency in $\beta$-gal ${ }^{-} /$neur ${ }^{-}$fibroblasts but had no effect on neuraminidase levels. These results agree with those of Suzuki et al. (15), but differ from those of Galjaard et al. (5) who reported leupeptin to be effective in correcting both $\beta$-galactosidase and neuraminidase. Our results suggest that $\beta$-galactosidase is rapidly degraded by proteases in the lysosomes of $\beta$-gal ${ }^{-} /$neur $^{-}$cells but that the addition of protease inhibitor leupeptin inhibits the process and allows $\beta$-galactosidase levels to increase. The absence of an effect of leupeptin on neuraminidase levels could have at least two explanations: 1 ) the accelerated neuraminidase turnover may be mediated by a protease which is not sensitive to leupeptin or 2) the missing "corrective factor" may be necessary not only to stabilize neuraminidase but also required for "activation" of its catalytic activity. In this case, leupeptin would not be expected to replace the "corrective factor." Overall our resuits are consistent with those recently published by d'Azzo et al. (3) and provide further evidence that combined $\beta$-galactosidase neuraminidase deficiency is not the result of a defect in the structural gene for either $\beta$-galactosidase or neuraminidase but is due to a defect in a glycoprotein factor that is required to protect these enzymes from degradation and allow their expression in lysosomes. The phenotypic correction of $\beta$-galactosidase by leupeptin and "corrective factor" suggests two approaches to therapy, at least for the $\beta$-galactosidase component of this disorder.

\section{REFERENCES AND NOTES}

1. Andria, G., Strisciuglio, P., Pontarelli, G., Sly, W. S., and Dodson, W. E. Infantile neuraminidase and $\beta$-galactosidase deficiencies (galactosialidosis) with mild clinical courses. In: G. Tettamanti, P. Durand, S. di Donato: Sialidosis and Sialidases. p. 379 (Ermes, Milano, 1981).

2. Brot, F. E., Glaser, J. H., Roozen, K. J., and Sly, W. S.: In vitro correction of deficient human fibroblasts by $\beta$-galactosidase from different human sources. Biochem. Biophys. Res. Commun. 57: 1 (1974).

3. d'Azzo, A.. Hoogeveen. A., Reuser. A. J. J.. Robinson, D., and Galjaard. H. Molecular defect in combined $\beta$-galactosidase and neuraminidase deficiency in man. Proc. Natl. Acad. Sci. U.S.A. 79: 4535 (1982).

4. Frisch, A. and Neufeld, E. F.: A rapid and sensitive assav for neuraminidase: Application to cultured fibroblasts. Anal. Biochem., 95: 222 (1979).

5. Galjaard, H. Hoogeveen. A., Verheijen, F., van Diggelen. O. P.. Konings, A. d'Azzo. A. and Reuser. A. J. J.: Relationship between clinical. biochemical and genetic heterogeneity in sialidase deficiency. In: G. Tettamanti. $P$. Durand. S. di Donato: Sialidoses and Sialidases. p. 317 (Ermes. Milano. 1981).

6. Glaser, J. H. and Sly. W. S.: B-Glucuronidase deficiency and mucopolysaccharidosis. Methods for enzymatic diagnosis. J. Lab. Clin. Med., 82: 969 (1973).

7. Gonzalez-Noricga. A., Grubb. J. H.. Talkad, V., and Sly, W. S.: Chloroquine inhibits lysosomal enzyme pinocytosis and enhances lysosomal enzyme secretion by impairing receptor recycling. J. Cell Biol., 85: 839 (1980).

8. Hoogeveen. A., d'Azzo. A., Brossmer, R., and Galjaard. H.: Correction of combined $\beta$-galactosidase/neuraminidase deficiency in human fibroblasts. Biochem. Biophys. Res. Commun., 103: 292 (1981).

9. Hoogeveen, A. T., Verheijen. F. W.. d’Azzo, A., and Galjaard, H.: Genetic heterogeneity in human neuraminidase deficiency. Nature. 285: 500 (1980).

10. Kato. T. Okada, S., Yutuka. T. Inui, K.. Yabuuchi. H.. Chivo. H.. Furuyama, $\mathrm{J}$.. and Okada, Y.: Beta-galactosidase deficient-type mucolipidosis: a complementation study of ncuraminidase in somatic cell hybrids. Biochem. Biophys. Res. Commun.. 91: 114 (1979).

11. Lowry. O. H.. Rosebrough. N. J., Farr. A. L.. and Randall, R. J.: Protein measurement with the folin phenol reagent. J. Biol. Chem., 193:265 (1951).

12. Miller. A. L.. Frost. R. G., and O'Brien. J. S.: Purification of human liver acid B-D-galactosidases using affinity chromatography. Anal. Biochem., 74: 537 (1976).

13. Mueller. O. T. and Shows. T. B.: Human $\beta$-galactosidase and $\alpha$-neuraminidase deficient mucolipidosis: genetic complementation and analysis of the neuraminidase deficiency. Hum. Genet.. 60: 158 (1982).

14. Suzuki. Y.. Nakamura, N.. Fukuoka, K.. Shimada, Y., and Uono, M.: $\beta-$ Galactosidase deficiency in juvenile and adult patients. Hum. Genet.. 36: 219 (1977).

15. Suruki, Y. Sakuraba. H., Hayashi, K.. Suzuki, K.. and Imahori, K.: $\beta$ Galactosidase-neuraminidase deficiency: restoration of $\beta$-galactosidase activity by protease inhibitors. J. Biochem. 90: 271 (1981).

16. Wenger. D. A., Tarby, T. J., and Wharton, C.: Macular cherry-red spot and myoclonus with dementia: coexistent neuraminidase and $\beta$-galactosidase deficiencies. Biochem. Biophys. Res. Commun. 82:584 (1978).

17. Requests for reprints should be addressed to: William S. Sly. M.D., Division of Medical Genetics. Department of Pediatries. St. Louis Children's Hospital. 500 So. Kingshighway, P.O. Box 14871. St. Louis, MO 63178 (USA)

18. This research was supported by United States Public Health Service Grant GM 21096 and the Ranken Jordan Trust Fund for the Crippling Diseases of Children.

19. Received for publication September 13, 1982.

20. Accepted for publication May 20,1983 